\title{
Obstetric outcome in pregnancy complicated by ovarian cysts
}

\author{
Sheela S. R. ${ }^{1}$, Sreeramulu P. N. ${ }^{2}$, Poonguzhali L. ${ }^{1 *}$, Arulselvi K. ${ }^{1}$
}

${ }^{1}$ Department of Obstetrics and Gynaecology, ${ }^{2}$ Department of Surgery, Sri Devraj Urs Academy of Higher Education and Research, Kolar, Karnataka, India

Received: 31 August 2017

Revised: 19 September 2017

Accepted: 25 September 2017

\author{
*Correspondence: \\ Dr. Poonguzhali L., \\ E-mail: sw_81989@hotmail.com
}

Copyright: (c) the author(s), publisher and licensee Medip Academy. This is an open-access article distributed under the terms of the Creative Commons Attribution Non-Commercial License, which permits unrestricted non-commercial use, distribution, and reproduction in any medium, provided the original work is properly cited.

\begin{abstract}
Background: Ovarian masses are diagnosed in $0.5-1 \%$ pregnancies. During pregnancy ovarian cysts can undergo: resolution of the cyst, change of ultrasound pattern, occurrence of ovarian torsion and intra-cystic haemorrhage or rupture. Ovarian masses (esp torsion) is a cause of pain abdomen during pregnancy. The choice of treatment is mainly conservative, provided the patient is asymptomatic. Dilemma in management arises when the patient is symptomatic. Optimal timing for a planned surgery is the second trimester as it is shown to have least neonatal outcome. The objective of this study was to evaluate management options for ovarian cyst in pregnancy and its effect on outcome of pregnancy.

Methods: This study was conducted for 2 years from June 2014 to June 2016, at RL Jalappa Hospital, Kolar. A total of 46 pregnant women were included. The pregnancy outcome and the management used were studied. Also studied were the complications most likely to occur in pregnancies complicated by ovarian masses ovarian masses.

Results: Out of 46 patients, $2(4.3 \%)$ patients with ovarian cyst torsion underwent emergency laparotomy. 8 patients underwent surgery $\left(6\right.$ in $2^{\text {nd }}$ trimester and 2 at term) for various complications. Only one patient had miscarriage and remaining patients continued till term. Histopathological report of all the masses excised were obtained and 6 were reported to be benign serous cystadenomas, 1 benign mucinous cystadenoma and 3 simple cysts.

Conclusions: Optimal management for ovarian cyst is conservative in pregnancy provided patient remain asymptomatic and characteristic of cyst are consistent with benign pathology. Surgical management is to be reserved for symptomatic patient.
\end{abstract}

Keywords: Histology, Ovarian cyst, Ultrasound

\section{INTRODUCTION}

Ovarian masses are diagnosed in $0.5-1 \%$ pregnancies. ${ }^{1}$ Ovarian tumours first diagnosed during pregnancy often present a challenge for both the obstetrician providing pregnancy care and for the consulting subspecialists. ${ }^{2}$ Although the vast majority of these tumours are benign, on rare occasions, patients present with tumours that turn out to be malignant requiring more comprehensive and extensive surgical procedures that are more likely to lead to pregnancy loss. Hence accurate knowledge of tumor characteristics, especially the ultrasound appearance and gestational age at diagnosis, are key prerequisite for establishing the most effective management plan not just for the index but also for future pregnancies. The most common benign organic ovarian tumours during pregnancy are dermoid cysts followed by Cystadenomas.

During pregnancy ovarian cysts can undergo the following changes: resolution of the cyst, change of ultrasound pattern, occurrence of ovarian torsion and intra-cystic haemorrhage which can adversely affect the pregnancy outcomes. ${ }^{3}$ Torsion of ovary if one such complication which is a surgical emergency. The risk of 
ovarian torsion rises by 5-fold during pregnancy. Incidence is 5 per 10,000 pregnancies. $^{4}$ The rate of ovarian torsion in women presenting to early pregnancy is $0.2 \% .^{5}$ Torsion of ovary is the total or partial rotation of the adnexa around its vascular axis or pedicle. Moderate size, free mobility and long pedicle are predisposing factors. The exact etiology is obscure. ${ }^{6}$ Complete torsion causes venous and lymphatic blockade leading to stasis and venous congestion, haemorrhage and necrosis. The cyst becomes tense and may rupture. Patient usually presents with acute severe pain abdomen and pelvic examination may reveal a tender cystic mass separate from the uterus. ${ }^{7}$

Therefore, it is important to individualise the line of treatment of every patient presenting with ovarian mass depending on the presenting complaints, size of the mass, benign or malignant pathology.

\section{METHODS}

Prospective observational study conducted int the Department of Obstetrics and Gynecology at R L Jalappa Hospital and Research Centre.

The duration of the study was 2 years from June 2014 to June 2016. A total of 46 were diagnosed as pregnancy with ovarian cyst. Out of these $40(87 \%)$ were diagnosed during pregnancy by routine ultrasonography, $4(8.7 \%)$ were diagnosed before pregnancy and 2 cases $(4.3 \%)$ presented at Emergency Department with ovarian cyst torsion.

All relevant maternal demographic was documented. Complications arising due to the ovarian cyst were evaluated. Pregnancy outcome in form of miscarriage, mode of delivery (vaginal delivery and caesarean section) were noted. All lesions managed surgically were sent for histopathology.

The surgical reports were reviewed for the description of the location, size, and the possible torsion of the cyst. The histopathological examination of the cyst for any loculations, presence of any papillary projections or any solid areas was performed.

\section{RESULTS}

Out of the 46 patients included in the study, maternal age in the study group ranged from 20-35 years (Table 1). $43.5 \%$ were primigravida and $56.5 \%$ were multigravida (Table 2). At the time of presentation $54.3 \%$ were in first trimester, $34.8 \%$ in second trimester and $10.9 \%$ in third trimester (Table 3). On ultrasound, there was evidence of medium/large well-defined septated cyst with multiple solid components and echogenic contents with no significant vascularity and some ovarian cysts were found to be simple cyst with clear contents, but no cysts were diagnosed as malignant ultrasonographically (Table 4).
Table 1: Distribution of maternal age.

\begin{tabular}{|ll|}
\hline Age (years) & $\mathrm{n}=\mathbf{4 6}$ \\
\hline $20-24$ & $24(52.2 \%)$ \\
\hline $25-29$ & $14(30.4 \%)$ \\
\hline $30-34$ & $8(17.4 \%)$ \\
\hline
\end{tabular}

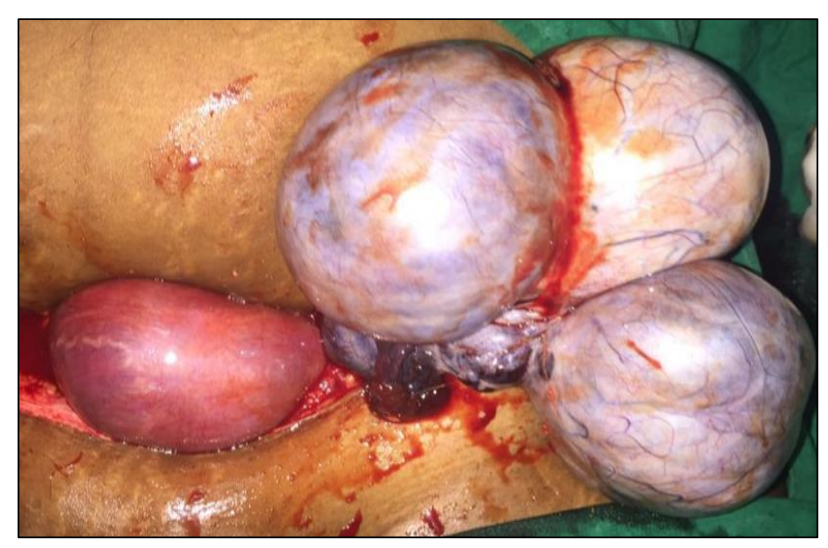

Figure 1: Torsion of left ovarian cyst in pregnancy (presented at 12 weeks of gestation).

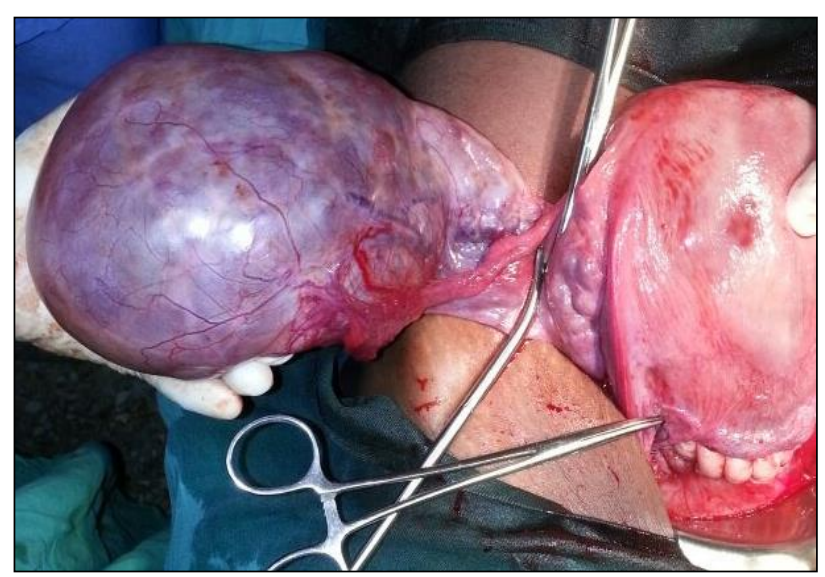

Figure 2: Ovarian cyst exteriorised after extraction of baby and closure of uterus.

Out of two patients with torsion of ovarian cyst who underwent emergency laparotomy, one patient delivered vaginally at term without maternal or foetal complications (Figure 1 and 2). The other patient came with PPROM at 25 weeks of gestation and pregnancy was terminated.

Table 2: Distribution of study group based on obstetric index.

\begin{tabular}{|ll|}
\hline Gravida & $\mathrm{N}=\mathbf{4 6}$ \\
\hline Primigravida & $20(43.5 \%)$ \\
\hline Multigravida & $26(56.5 \%)$ \\
\hline
\end{tabular}

Remaining 44 patients were managed conservatively (cyst of size $<5 \times 5 \mathrm{~cm}$ ) out of which 2 patients were hospitalized due to pain abdomen and discharged after 
pain relief, $6(13 \%)$ patients had elective laparotomy in second trimester due to increasing size (mean size of cyst was $10.5 \times 10.2 \mathrm{~cm}$ ) and no relief of pain and $2(4.3 \%)$ patients underwent caesarean section along with cystectomy (mean size of cyst was $15 \times 12.5 \mathrm{~cm}$ ) at term (Table 5). Only one pregnancy ended into miscarriage and remaining patients had a term delivery, the mode of delivery being vaginal in most cases (28 out of 46) (Table $6)$.

Table 3: Distribution of study group based on gestational age at time of presentation.

\begin{tabular}{|ll|}
\hline Trimester & $\mathbf{N}=46$ \\
\hline First (up to 12 weeks) & $25(54.3 \%)$ \\
\hline Second (13-28 weeks) & $16(34.8 \%)$ \\
\hline Third (29-40 weeks) & $5(10.9 \%)$ \\
\hline
\end{tabular}

Table 4: Distribution based on ultrasound features.

\begin{tabular}{|ll|}
\hline Ultrasound features & $\mathbf{n = 4 6}$ \\
\hline Benign features & $46(100 \%)$ \\
\hline Malignant features & 0 \\
\hline
\end{tabular}

Table 5: Complications of ovarian cysts.

\begin{tabular}{|ll|}
\hline Complications of ovarian cyst & $\mathbf{N}=46$ \\
\hline Torsion & $2(4.3 \%)$ \\
\hline Rupture & 0 \\
\hline Haemorrhage & 0 \\
\hline $\begin{array}{l}\text { Abdominal surgery during pregnancy } \\
\text { (due to increase in size and pain) }\end{array}$ & $6(13 \%)$ \\
\hline Hospitalization due to pain & $2(4.3 \%)$ \\
\hline
\end{tabular}

Table 6: Pregnancy outcome.

\begin{tabular}{|ll|}
\hline Obstetric outcome & $\mathbf{N}=46$ \\
\hline Vaginal delivery & $28(60.9 \%)$ \\
\hline Caesarean section & $17(37 \%)$ \\
\hline Miscarriage & $1(2.1 \%)$ \\
\hline
\end{tabular}

The lesions of 10 patients who underwent surgery were sent for histopathology and 6 lesions were benign serous cystadenomas and rest were benign mucinous cystadenoma and simple cysts.

\section{DISCUSSION}

Several surgical and/or medical emergencies/urgencies may occur in pregnant women. Pain abdomen is one of the most common emergencies that women present with during pregnancy. Particularly, ectopic pregnancies, ruptured or hemorrhagic ovarian cysts, ovarian or adnexal torsions, threatened or inevitable miscarriages, complications involving the uterine fibroids and spontaneous uterine rupture are possible acute complications.
Classically, the patient comes to the observation because of acute pelvic and/or abdominal pain, with or without vaginal bleeding or discharge. The diagnosis is suspected on the basis of symptoms, and made by means of physical evaluation, including abdominal, pelvic, and bimanual gynecological examinations, of transvaginal (TVS) and/or transabdominal (TAS) sonography, and laboratory tests. ${ }^{8}$

Adnexal torsion is considered a true gynecologic emergency. It may involve ovary, fallopian tube, or both and is due to partial or complete twisting of the vascular pedicle, which results venous, arterial, and lymphatic obstruction until ovarian necrosis. The right adnexa is most commonly involved, probably because of the longer utero-ovarian ligament than the left, and the resulting hypermobility. The left adnexa is less mobile because of the presence of the sigmoid colon, which reduces the space needed for torsion. Adnexal torsion can occur at any age. It is estimated that $70-80 \%$ of adnexal torsions occur in women of reproductive age, and 12-25\% of women with torsion are pregnant. It is commonly associated with an increase of the ovarian size and weight. ${ }^{9}$

Laparoscopy is currently the most common surgical approach in the management of ovarian cysts. ${ }^{10}$

In a study done by Savelli et al, 11 out of total 50 patients, 35 patients had simple ovarian cysts, 8 had cystadenofibroma, 5 had cystadenomas, and 2 had serous papillary borderline tumors.

Smorgick et al, 12 reported 7 cystadenomas and 8 cystadenofibromas out of 47 ovarian cysts on histopathology.

Previous studies have found that dermoid cyst is the most common diagnosis in the case of benign ovarian cyst in pregnancy. ${ }^{13}$ However, the present study found that serous cyst adenoma is the most common diagnosis among the pregnant women with ovarian cysts.

\section{CONCLUSION}

Optimal management for ovarian cyst is conservative in pregnancy provided patient remain asymptomatic and characteristic of cyst are consistent with benign pathology. Surgical management is to be reserved for symptomatic patient.

\section{Funding: No funding sources \\ Conflict of interest: None declared}

Ethical approval: The study was approved by the Institutional Ethics Committee

\section{REFERENCES}

1. Mascilini F, Savelli L, Scifo MC, Exacoustos C, Timor-Tritsch I, De Blasis I, et al. Ovarian masses 
with papillary projections diagnosed and removed during pregnancy: ultrasound features and histological diagnoses. Ultrasound Obstet Gynecol. 2016;30:230-4.

2. Husseinzadeh N, Sibai B, Siddiqi TA. Ovarian tumors in pregnancy: diagnosis and management. Am J Perinatol. 2012;29:327-34.

3. Tariel O, Huissoud C, Rudigoz RC, Dubernard G. Benign-appearing ovarian tumors during pregnancy. Gynecol J Obstet Reproductive Biol. 2013;842-55.

4. Damigos E, Johns J, Ross J. An update on the diagnosis and management of ovarian torsion. Obstet Gynecol. 2012;14:229-36.

5. Oelsner G, Shashar D. Adnexal torsion. Clin Obstet Gynecol. 2006;49:459-63.

6. Ventolini G, Hunter L, Drollinger D, Hurd WW. Ovarian torsion during pregnancy. Resident and Staff Physician. 2005;51(9):26.

7. Lee CH, Raman S, Sivanesaratnam V. Torsion of ovarian tumors: a clinicopathological study. Int J Gynaecol Obstet. 1989;28:21-5.

8. Levine D, Brown DL, Andreotti RF, Benacerraf B, Benson CB, Brewster WR, et al. Management of asymptomatic ovarian and other adnexal cysts imaged at US: Society of radiologists in ultrasound consensus conference statement. Radiol. 2010;256:943-54.
9. Hursitoglu BS, Demirtas GS, Demitras O, Alman L, Yilmaz H. A clinic-pathological evaluation of 194 patients with ovarian teratoma: 7-year experience in a single centre. Ginekologia Polska. 2013;84:108-11.

10. Darwish AM, Amin AF, Mohammad SA. Laparoscopic management of paratubal and paraovarian cysts. JSLS. 2003;7:101-6.

11. Savelli L, Ghi T, De Iaco P, Ceccaroni M, Venturoli S, Cacciatore B. Paraovarian/paratubal cysts: Comparison of transvaginal sonographic and pathological findings to establish diagnostic criteria. Ultrasound Obstet Gynecol. 2006;28:330-4.

12. Smorgick N, Herman A, Schneider D, Halperin R, Pansky M. Paraovarian cysts of neoplastic origin are underreported. JSLS. 2009;13:22-6.

13. Schmeler KM, Mayo-Smith WW, Peipert JF, Weitzen S, Manuel MD, Gordinier ME. Adenexal masses in pregnancy: surgery compared with observation. Obstet Gynecol. 2005;105:1098-103.

Cite this article as: Sheela SR, Sreeramulu PN, Poonguzhali L, Arulselvi K. Obstetric outcome in pregnancy complicated by ovarian cysts. Int J Reprod Contracept Obstet Gynecol 2017;6:5051-4. 